\title{
Multifractality in China copper futures market
}

\author{
Bai Manying ${ }^{1, a}$, Guo Fengjuan ${ }^{2, b}$ \\ ${ }^{1,2}$ School of Economics and Management, Beihang University, No. 37, Xueyuan Road, Haidian \\ District, Beijing, 100191, China \\ aemail: baimy805@gmail.combemail: beihangpass@163.com
}

Keywords: Multifractality; China Copper futures market; MFDFA; Sliding window technology

\begin{abstract}
We use MFDFA method in this study to examine the multifractality of China copper futures market from 2006 to 2012 for the Shanghai Futures Exchange (SFE). Results show that China copper futures market displays multifractal scaling behavior. We also find that the copper futures price fluctuation on SFE is intensive. However, the SFE is more likely to have some profit. The results can provide important implications for understanding the nature of China futures market.
\end{abstract}

\section{Introduction}

The study of finanicial market's variation rule has already received a great deal of attentions. Much research has been done in nonlinear science and corresponding complexity for the last few years, ranging from soliton to chaos to fractal [1-4]. MFDFA is widely used to analyse long-range autocorrelations and capture the volatility statistical characteristic in different time scale for non-stationary time series..

However, these MFDFA method [5] will cause new pseudo-wave error due to the discontinuity in segmentation join points during the polynomial fitting, which will lead to distortion of the scaling index [6]. In our paper, we propose a S-R-MFDFA method, which uses the continuous overlapping interval segmentation method to replace the non-overlapping interval segmentation method in the R-MFDFA method and can reduce pseudo-wave error significantly.The S-R-MFDFA method is applied to the copper futures return series of Shanghai Futures Exchange (SFE) and London Metal Exchange (LME) for empirical research. We validate the multifractality of copper futures returns in SFE and LME. Additionally, the degree of multifractality and the market risk is analyzed and compared for the two markets.

\section{Our approach}

We use the MFDFA approach for detecting multifractality of time series. For time series $\left\{x_{k}, k=1,2, \cdots, N\right\}$, the MFDFA method can be summarized with the following steps.

(1) Construct a new series and calculate:

$$
y(i)=\sum_{k=1}^{i}\left[x_{k}-<x>\right](i=1,2, \cdots, N)
$$

(2) The new series $y(i)$ is divided into $N_{t}\left(N_{t}=\operatorname{int}(N /(s-1))\right)$ non-overlapping subtime series of $s$ sliding window lengths (The overlap length is 1 ).

(3) For each subtime series $v\left(v=1,2, \cdots, 2 N_{t}\right)$, there is $s$ points for $v$. We adopt the polynomial regression and obtain local trend function $\tilde{y}(v)$.

(4) We calculate the average variance for each segments.

$$
\begin{aligned}
& F^{2}(v, s)=\frac{1}{s} \sum_{i=1}^{s}\{y[(v-1) s+i]-\tilde{y}(i)\}^{2}\left(v=1,2, \cdots, N_{t}\right) \\
& F^{2}(v, s)=\frac{1}{s} \sum_{i=1}^{s}\left\{y\left[N-\left(v-N_{t}\right) s+i\right]-\tilde{y}(i)\right\}^{2}\left(v=N_{t}+1, N_{t}+2, \cdots, 2 N_{t}\right)
\end{aligned}
$$


(5) Determine the $q$-order fluctuation function by the following formula:

$F_{q}(s)=\left\{\frac{1}{2 N_{t}} \sum_{v=1}^{2 N_{t}}\left[F^{2}(v, s)\right]^{q / 2}\right\}^{1 / q}, \quad q \neq 0$

$F_{0}(s)=\exp \left\{\frac{1}{4 N_{t}} \sum_{v=1}^{2 N_{t}} \ln \left[F^{2}(v, s)\right]\right\}, \quad q=0$

(6) Calculate the corresponding fluctuation function $F_{q}(s)$ of different $s$. For time series, $F_{q}(s)$ and $s$ satisfies the scaling or power-law relationship: $F_{q}(s) \propto s^{h(q)}$

Here, the scaling exponent $h(q)$ is one nonlinear function. If $h(q)$ is the function of order number $q$, time series $\left\{x_{k}\right\}$ is multifractal process. The Renyi exponent $\tau(q)$ is a nonlinear function of $q$. The multifractal spectrum $f(\alpha)$ is the quantitative index of multifractal behavior, and $\alpha$ is the singular exponent which describes the fractal feature of each part (time-point) in the whole process.

\section{Multifractality analysis of copper futures price}

\subsection{Data set}

In this study, we choose the daily closing price series of copper futures on the Shanghai Futures Exchange (SFE) as data set to represent the Shanghai copper futures markets. The period of the data set ranges from 1 January 2006 to 6 April 2012. The size of SFE data is 1506.

\subsection{Multifractal analysis}

We use the MFDFA to analyze the copper futures price of SFE. From Fig. 1, we can find that,

(1) When the $q$-order increases from -10 to 10 , the generalized Hurst exponents $h(q)$ show a decreasing trend, which means that the $h(q)$ is obviously not a constant and depends on $q$. It indicates that the time series of log return rate are apparently multifractal in SFE.

(2) For $q=2$, the values of Hurst index $h(q)$ of SFE are larger than 0.5, which means that the time series of log return rate of the copper market present positive long-range correlation.

(3) For negative or small positive $q$, the generalized Hurst exponents $h(q)>0.5$. It indicates that the influence of small fluctuations of copper futures return series is magnified, which presents the persistence of return series and highlights the role of intrinsic factor in market. For large positive $q, h(q)<0.5$. It implies that large fluctuations are dominant and the return series presents the sustainability. It is probably due to China government's macroeconomic regulation and control.

(4) For the fluctuation degree of $\Delta h(\Delta h(q)=\max (h(q))-\min (h(q))), \sigma(h)$ and $\Delta \alpha$, we can conclude that the values of $\Delta h, \sigma(h)$ and $\Delta \alpha$ are large on SFE. Hence the multifractality of SFE is strong.

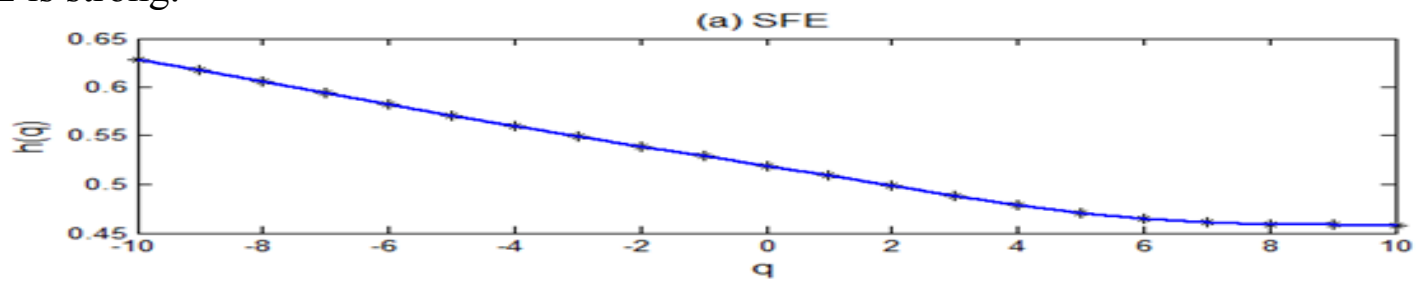

Fig. 1 Plots of $h(q)$ versus $q(q=-10,-9,-8, \cdots, 8,9,10)$ 


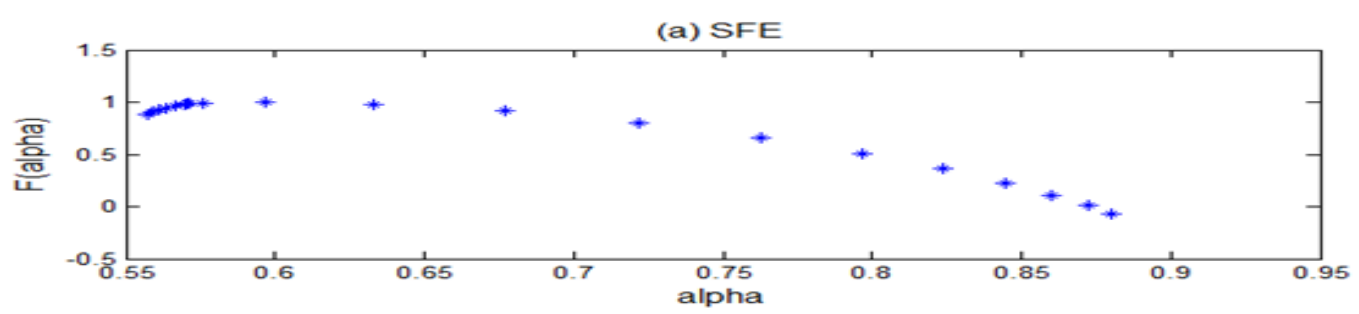

Fig. 2 Plots of singular general function $f(\alpha)$ versus $\alpha$ for SFE

Fig. 2 plots the multifractal spectra $f(\alpha)$ versus $\alpha$. The $\Delta f\left(\Delta f=f\left(\alpha_{\min }\right)-\left(\alpha_{\max }\right)\right)$ indicates the copper futures price reaches the highest points more frequently than the lowest points. The $\Delta f$ value of SFE is large, which implies the copper futures of SFE have a lager chance to make profit in general.

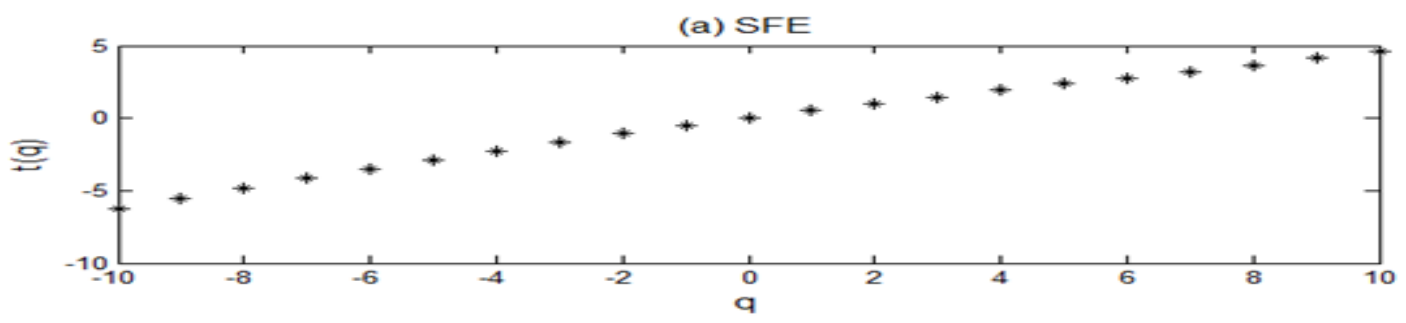

Fig. 3 Plots of scaling exponent function $\tau(q)$ versus $q$ for SFE

Form Fig. 3, we can see that the multifractal scaling exponent function $\tau(q)$ of the logarithmic return series has a non-linear relationship with $\mathrm{q}$ and is a convex function of monotonous growth. The observation confirms the multifractality of the return series of copper futures.

\section{Conclusion}

We first applied the MFDFA method in this paper to investigate multifractality in the copper futures markets of SFE. Our result are summarized as follows:

First, the copper futures market of SFE shows multifractal scaling behavior. Second, for a given $q$, when $q$ is positive and relatively large, the generalized Hurst exponents $h(q)$ for SFE is large, which illustrates that the long-range correlation of the copper futures return series $r$ is strong.

From the analysis of the multifractality of copper futures return series, we find that the fluctuation of copper futures return series presents the nonlinear change, which shows inherent correlation behind the randomness and uncertainty. The inherent long-range correlation implies that the fluctuation of copper futures return series is caused not only simply by the outside disturbance of the random factors but also the complex nonlinear relationship, which may mainly comes from the progressive process of fluctuations.

\section{Acknowledgements}

This work is supported by the National Science Foundation of China under Grant No. 71071009 .

\section{References}

[1] Peters E. Fractal market analysis: applying chaos theory to investment and economics [M]. New York: John Wiley \& Sons, 1994.

[2] Hurst H E. Long term storage capacity of reservoirs [ J ]. Transactions American Society of Civil Engineers, 1951, (116): 770 - 808.

[3] Peng C K, Buldyrev S V, Havlin S 1994 Phys. Rev. E 491685.

[4] Kantelhardt J W, Zschiegner S A, Koscielny B E, et al. Multifractal detrended fluctuation analysis of nonstationary timeseries [ J ]. Physica A, 2002, 316: 87 -114. 
[5] Kantelhardt J W, Zschiegner S A, Koscielny-Bund Eva, et al. Multifracta detrended fluctuation analysis of nonstationary time series [J]. Physica A, 2002, 316: 87-114.

[6] $\mathrm{Xu}$ Shufang, The theory and method of matrix calculation [M]. Beijing: Peking University Press, 1995, 10-12. 